Hans Dirrigl, Dietmar Wellisch, Ekkehard Wenger (Hrsg.)

Steuern, Rechnungslegung und Kapitalmarkt 
GABLER EDITION WISSENSCHAFT 
Hans Dirrigl, Dietmar Wellisch,

Ekkehard Wenger (Hrsg.)

\section{Steuern, Rechnungslegung und Kapitalmarkt}

Festschrift für Franz W. Wagner

zum 60. Geburtstag

Deutscher Universitäts-Verlag 
Bibliografische Information Der Deutschen Bibliothek

Die Deutsche Bibliothek verzeichnet diese Publikation in der Deutschen Nationalbibliografie; detaillierte bibliografische Daten sind im Internet über $<$ http://dnb.ddb.de> abrufbar.

\section{Auflage Juni 2004}

Alle Rechte vorbehalten

(C) Deutscher Universitäts-Verlag/GWV Fachverlage GmbH, Wiesbaden 2004

\section{Lektorat: Ute Wrasmann}

Der Deutsche Universitäts-Verlag ist ein Unternehmen von Springer Science+Business Media.

www.duv.de

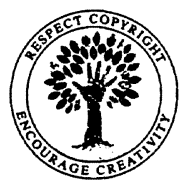

Das Werk einschließlich aller seiner Teile ist urheberrechtlich geschützt. Jede Verwertung außerhalb der engen Grenzen des Urheberrechtsgesetzes ist ohne Zustimmung des Verlags unzulässig und strafbar. Das gilt insbesondere für Vervielfältigungen, Übersetzungen, Mikroverfilmungen und die Einspeicherung und Verarbeitung in elektronischen Systemen.

Die Wiedergabe von Gebrauchsnamen, Handelsnamen, Warenbezeichnungen usw. in diesem Werk berechtigt auch ohne besondere Kennzeichnung nicht zu der Annahme, dass solche Namen im Sinne der Warenzeichen- und Markenschutz-Gesetzgebung als frei zu betrachten wären und daher von jedermann benutzt werden dürften.

Umschlaggestaltung: Regine Zimmer, Dipl.-Designerin, Frankfurt/Main Gedruckt auf säurefreiem und chlorfrei gebleichtem Papier

ISBN-13: 978-3-8244-8145-3

e-ISBN-13: 978-3-322-81787-7

DOI: $10.1007 / 978-3-322-81787-7$ 


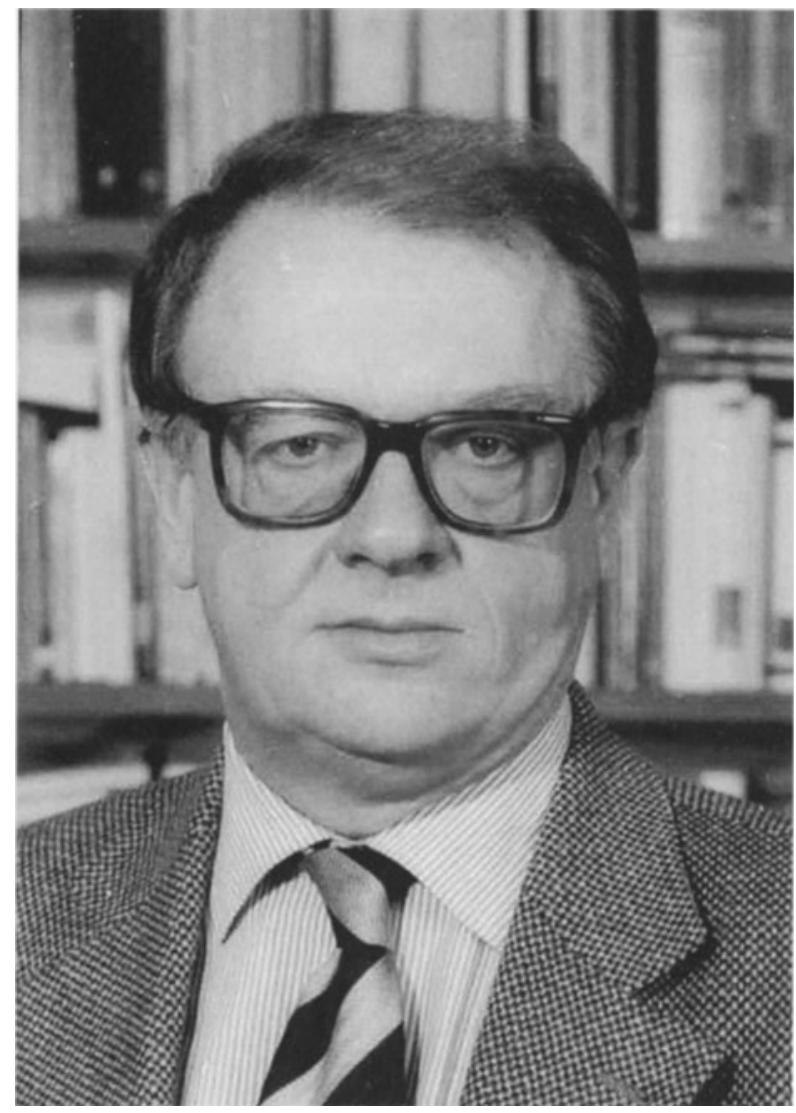




\section{Vorwort}

Franz W. Wagner begeht am 21. Mai 2004 seinen 60. Geburtstag. Wer ihn lange genug kennt, muss vermuten, dass er diesem Ereignis mit gemischten Gefühlten entgegensieht. Als Enddreißiger plagte ihn stets die Befürchtung, alsbald vom normalen Niedergang der wissenschaftlichen Originalität jenseits des 40 . Lebensjahres betroffen zu sein. Nachdem sich diese Befürchtung bislang als unbegründet erwiesen hat, wird er es uns nachsehen, dass wir sein wissenschaftliches Werk und unsere persönliche Verbundenheit schon jetzt zum Anlass nehmen, ihm diese Festschrift zu widmen.

Franz Wagner wurde 1944 in Újpetre, Ungarn, geboren. Danach erlebte er die ersten Jahre eines neu entstehenden Arbeiterparadieses in Sachsen. Vom dort erlangten Idiom macht er bei Bedarf auch heute noch in einer Weise Gebrauch, als wäre er nie weggegangen. Wie er die sonstigen Erfahrungen aus dieser Zeit verarbeitet hat, war nicht nur seinen Berichten über einen Besuch der „Zone“ in den späten 80er Jahren zu entnehmen, sondern hat sich auch in einer Grundeinstellung zu Fragen der Wirtschaftsordnung niedergeschlagen, die man bei Lektüre seiner wissenschaftlichen Veröffentlichungen nicht lange suchen muss. Nachdem er den Segnungen des sächsischen Sozialismus gerade noch rechtzeitig entkommen war, definierte er seine landsmannschaftliche Identität schließlich als Bewohner des Freistaates Bayern, wo er in Freising im Jahre 1964 die Reifeprüfung ablegte und sich danach einem Studium der Betriebswirtschaftslehre an der Ludwig-Maximilians-Universität in München zuwendete. Mit dem Examen erreichte er 1969 ein akademisches Zwischenziel. Bereits zwei Jahre später erfolgte an der staatwirtschaftlichen Fakultät der Ludwig-Maximlians-Universität die Promotion zum Dr. oec. publ. Seine Dissertation trug den Titel „Das Ausscheiden eines Gesellschafters aus einer OHG - Ein Beitrag zur Theorie der Unternehmensbewertung“. Anschließend setzte er seine Laufbahn an der wirtschaftswissenschaftlichen Fakultät der Universität Regensburg fort. Dort fand er eine neue Einstellung zur Betriebswirtschaftslehre als wissenschaftlicher Disziplin und habilitierte sich im Jahre 1976 mit einer Arbeit über: „Kapitalerhaltung, Geldentwertung und Gewinnbesteuerung“. Bereits diese Schrift belegt das außerordentliche Interesse Franz Wagners an konzeptionellen Fragen der Unternehmensrechnung und Besteuerung, die bis heute im Zentrum seines Schaffens stehen.

Unmittelbar nach der Habilitation wurde Franz Wagner auf den Lehrstuhl für Betriebswirtschaftliche Steuerlehre einschließlich Revisions- und Treuhandwesen an der Universität Hohenheim in Stuttgart berufen. Zwei der Herausgeber haben seine Hohenheimer Phase zumindest zeitweise begleitet. Im Jahre 1982 erhielt Franz Wagner einen Ruf an die Universität Hamburg, den er aber ablehnte. Erst vier Jahre später hielt Franz Wagner seine Mission in Hohenheim für nicht weiter fortsetzungsbedürftig und nahm im Jahre 1986 einen Ruf auf den Lehrstuhl für Betriebswirtschaftliche Steuerlehre und Wirtschaftsprüfung an der EberhardKarls-Universität Tübingen an, wo er bis heute geblieben ist. Einen Ruf nach Würzburg im Jahre 1994, der ihm die Rückkehr ins geliebte Bayern ermöglicht hätte, hat er am Ende ebenso wenig angenommen wie einen nahezu zeitgleich eingetroffenen Ruf nach Wien. Mehrfach hat er dort allerdings Gastprofessuren wahrgenommen und ist deshalb im Jahre 2001 zum Honorarprofessor an der Fakultät für Wirtschaftswissenschaften und Informatik der Universi- 
tät Wien ernannt worden. Bereits ein Jahr zuvor hat ihm die aus der alten staatswirtschaftlichen Fakultät hervorgegangene und seither grundlegend umbesetzte Fakultät für Betriebswirtschaftslehre der Ludwig-Maximilians-Universität München die Würde eines Ehrendoktors verliehen.

Franz Wagners engagiertes Wirken in der Kommission „Betriebswirtschaftliche Steuerlehre" des Verbandes der Hochschullehrer für Betriebswirtschaft, deren Vorsitz er von 1987 bis 1989 bekleidete, lässt sein intensives Interesse am Forschungsprogramm der Betriebswirtschaftlichen Steuerlehre erkennen. Von deren betriebswirtschaftlicher Verortung ausgehend, ist es ihm ein persönliches Anliegen, die Verbindung zu Steuerrechts- und Finanzwissenschaftlern zu pflegen. Seinem Wirken im Ausschuss Unternehmensrechnung des Vereins für Socialpolitik, dessen Vorsitz er von 1991 bis 1995 innehatte, ist es maßgeblich zu verdanken, dass dieser Kreis um Mitglieder aus dem Bereich der Rechtswissenschaften erweitert wurde, um auf diese Weise dem gesamten Spektrum der Unternehmensrechnung und Steuerwissenschaften eine breite Plattform für fruchtbare Diskussionen bieten zu können. Das gleiche Anliegen unterstützt Franz Wagner auch dadurch, dass er Mitherausgeber der Zeitschrift „Steuer und Wirtschaft" ist.

Als akademischer Lehrer wird Franz Wagner den bereits nach Tausenden zählenden Zuhörern seiner Vorlesungen auch noch nach Jahren in bleibender und lebhafter Erinnerung sein, nicht nur wegen der fachlichen Inhalte, sondern auch wegen der unvergleichlichen Art der Darbietung. Die Fähigkeit zur kritischen Analyse ökonomischer Sachverhalte vermittelt er in einer Art und Weise, der auch im Unterhaltungswert eine Spitzenposition zukommt. Dies können auch diejenigen bestätigen, die bei vielen Vorträgen Franz Wagners außerhalb des universitären Bereichs in den Genuss des Zuhörens gekommen sind.

Seine Doktoranden wissen allerdings, dass es ihm stets um mehr geht. Franz Wagner wollte sich immer mit den von ihm betreuten Dissertationsschriften identifizieren können und seinen Doktoranden bestimmte wissenschaftliche Botschaften mitgeben. Seine konzeptionellen und inhaltlichen Ansprüche sind in der Praxis nicht immer sofort auf Verständnis gestoßen. Der Erfolg seiner Doktoranden in den verschiedensten Positionen der Wirtschaft verdeutlicht aber, dass ein hohes Niveau wissenschaftlicher Arbeiten nicht etwa mangels platter Praxisnähe eine Bewährung in der Praxis fraglich erscheinen lässt, sondern vielmehr eine Praxisbewährung fördert. Wer gründlich nachdenkt und argumentiert, fördert seine Aussichten, scheinbar bewährte Praktiken einer begründeten Revision zu unterziehen.

Einen ebenso hohen Stellenwert hatte für Franz Wagner die Sicherstellung qualifizierten wissenschaftlichen Nachwuchses, wobei man unter dessen Förderung nicht nur die Betreuung von Habilitationen verstehen darf. Die wegweisende Hilfestellung war nicht auf den Kreis seiner Lehrstuhlmitarbeiter beschränkt, sondern umfasste auch Personen, die zunächst nicht in einer unmittelbaren Verbindung zu ihm standen, wenn diese Förderung im Interesse der Fortentwicklung und wissenschaftlichen Profilierung seines Faches war. So unterstützte Franz Wagner neben vielen anderen Personen zwei der Herausgeber dieser Festschrift bei ihrer Integration in die wissenschaftliche Gemeinde, ohne dass es sich unmittelbar um Schüler im engeren Sinne handelt.

Die Förderung des wissenschaftlichen Nachwuchses ist aber nicht bloß über den direkten persönlichen Kontakt erfolgt, sondern auch über das Schriftum des Jubilars. Franz Wagner 
kann auf ein eindrucksvolles wissenschaftliches Werk zurückblicken. Dessen Würdigung soll den Verfassern der Beiträge dieser Festschrift vorbehalten bleiben, die das breite Spektrum der wissenschaftlichen Interessen aus den verschiedensten Blickwinkeln beleuchten.

Jenseits unterschiedlicher persönlicher Auffassungen zu fachlichen Einzelfragen muss hervorgehoben werden, dass sich Franz Wagner um die programmatische Ausrichtung des Fachs „Betriebswirtschaftliche Steuerlehre“ intensiv gekümmert, ja sich sogar dafür persönlich verantwortlich gefühlt hat. Dieses Unterfangen ist nicht einfach, da die betriebswirtschaftliche Steuerlehre den schwierigen Spagat zwischen akademischem Anspruch und steuerrechtlicher Praxisnähe zu bewältigen hat. Umso verdienstvoller ist es, sich immer wieder diesem Problem zuzuwenden und jüngere Kollegen dafür zu sensibilisieren.

Sein mit einem der Herausgeber zusammen verfasstes Buch zur Steuerplanung der Unternehmung hat nachhaltig die Entwicklung der betriebswirtschaftlichen Steuerlehre in Deutschland beeinflusst. Noch heute, gut 20 Jahre nach seinem Erscheinen, wird dieses Werk von allen jüngeren Wissenschaftlern, die sich intensiver mit Fragen der Steuerplanung beschäftigen, als Standardwerk im deutschen Sprachraum zitiert. An einer vergleichsweise umfassenden Neubearbeitung dieses Themas hat sich seither niemand mehr versucht. Die zunehmende Auflösung des Steuerrechts in einen unüberschaubaren Wirrwarr kasuistischer Einzelregelungen macht eine konzeptionelle Durchdringung des Sachverhalts heute praktisch unmöglich. Franz Wagners Wunsch wäre die gegenteilige Entwicklung gewesen: Ein systematisches, möglichst entscheidungsneutrales Steuerrecht, was eine Neuauflage seines Standardwerks nicht unmöglich, sondern überflüssig macht. Gerade seinen jüngsten Beiträgen ist zu entnehmen, dass dies auf etwas ganz anderes hinausläuft als die so gut gemeinten Reformvorschläge von selbst ernannten „Radikalreformern“ des Steuerrechts, die von Franz Wagner viel lernen könnten, insbesondere, dass ,gut gemeint" das Gegenteil von gut ist.

Über das Schrifttum haben sich Ausstrahlungseffekte bei den Fachvertretern ergeben, die erkennen lassen, dass sie beim Lehrmeister Franz Wagner auf indirekte Weise in der fachlichen Ausbildung gewesen sind. Man wird nicht übertreiben, wenn man behauptet, dass aus Schülern oft Jünger geworden sind, also „Wagnerianer“, die das wissenschaftliche Credo des Jubilars unbeirrt von modischen Erscheinungen unterstützen.

Die Herausgeber danken allen Autoren dieser Festschrift für ihre Mitarbeit und bitten bei all denen um Verständnis, die ihre Verbundenheit mit dem Jubilar nicht durch einen Beitrag zum Ausdruck bringen konnten. Unser besonderer Dank gilt Frau Ute Wrasmann, Cheflektorin für Wissenschaftliche Monographien des Deutschen Universitäts-Verlags, die sich ohne Zögern bereit erklärt hat, diese Festschrift zu verlegen. Wir danken auch Frau Birgitt C. Fehr, die die Manuskripte unter hohem Zeitdruck zusammengefasst und für eine weitgehend einheitliche Formatierung gesorgt hat. Ebenso sei unseren Mitarbeitern Frank Betz und David Stüker sowie den Mitarbeiterinnen und Mitarbeitern des Lehrstuhls für BWL, Bank- und Kreditwirtschaft der Universität Würzburg für ihren Einsatz bei Durchsicht der Manuskripte gedankt. Verbliebene Mängel haben natürlich allein die Herausgeber zu verantworten.

Die Festschrift ist den Themen Steuern, Rechnungslegung und Kapitalmarkt gewidmet. Da sich viele ihrer Beiträge diesen Bereichen nicht trennscharf zuordnen lassen, wurden sie in alphabetischer Autorenreihenfolge angeordnet. 
Wir wünschen Franz Wagner, dass ihm seine Gesundheit als wichtigste Voraussetzung für ein weiterhin profiliertes Eintreten für die Belange unseres Faches noch viele Jahre erhalten bleibt.

Hans Dirrigl

Dietmar Wellisch

Ekkehard Wenger 


\section{Inhaltsverzeichnis}

Vorwort der Herausgeber

Hans Dirrigl

Die Besteuerung in Kalkülen zur Unternehmensbewertung bei

Wachstum und Risiko

Hans Fehr und Wolfgang Wiegard

Abgeltungssteuer, duale ESt und zinsbereinigte ESt: Steuerreform

aus einem Guss

Christoph Kaserer und Leonhard Knoll

$\S 305$ Abs. 3 Satz 3 AktG: Iudex non calculat

Dirk Kiesewetter und Rainer Niemann

Die versehentliche Einführung einer konsumorientierten Einkommensteuer in Österreich und Lehren für die Abgeltungssteuer in Deutschland

Lutz Kruschwitz und Andreas Löffler

Mehr Fragen als Antworten im Zusammenhang mit Steuervorteilen

bei der Unternehmensbewertung

Hans-Ulrich Küpper

Cash Flow und/oder Vermögen als Basis von Unternehmensrechnung und Besteuerung?

Branka Lončarević und Manfred Rose

Vermeidung internationaler Doppelbesteuerung bei konsumorientierten

Einkommensteuern

Rolf Nonnenmacher

Value Reporting als Erweiterung der internationalen Rechnungslegung?

- Zur finanziellen Berichterstattung deutscher Unternehmen -

\section{Dieter Schneider}

Franz W. Wagners Analyse des Maßgutproblems in ihren Folgen

für Kapitalerhaltung, Geldentwertung und Gewinnbesteuerung

Ulrich Schreiber und Martin Ruf

Die Messung der effektiven Steuerbelastung. Ein Vergleich verschiedener effektiver Steuersätze 
Theodor Siegel

System der Einkommensteuer und Rechtsformneutralität

Jochen Sigloch

Unternehmenserfolgsbesteuerung zwischen Einkommen und Konsum -

Bestandsaufnahme und Anwendungsperspektiven

Hannes Streim, Marcus Bieker und Maik Esser

Der schleichende Abschied von der Ausschüttungsbilanz - Grundsätzliche

Überlegungen zum Inhalt einer Informationsbilanz

Dietmar Wellisch und Reiner Schwinger

Einzelbewertung oder korrespondierende Bewertung: Die Bilanzierung von Pensionszusagen mit Asset Funding

Ekkehard Wenger und Renate Hecker

Der Vorzusaktionär als bevorzugtes Plünderungsopfer 